Prepared for publication in IEEE Conference Proceedings. 29th International conference on infrared and millimeter waves and 12th International Conference on Terahertz Electronics

\title{
Terahertz Probes of Transient Conducting and Insulating Phases in Quasi-2D Electron-Hole Gases
}

\author{
R. A. Kaindl, D. Hägele*, M. A. Carnahan, R. Lövenich, and D. S. Chemla \\ Department of Physics, University of California at Berkeley and Materials Sciences Division, \\ E. O. Lawrence Berkeley National Laboratory, Berkeley, CA 94720, USA \\ e-mail: RAKaindl@lbl.gov
}

\begin{abstract}
We employ ultrafast terahertz ( $\mathrm{THz}$ ) pulses to study the dynamical interplay of optically-induced excitons and unbound electron-hole pairs in GaAs/AlGaAs quantum wells. A distinct low-energy oscillator appears upon resonant excitation of heavy-hole excitons, linked to transitions between their internal degrees of freedom. Time-resolving changes in the $\mathrm{THz}$ conductivity, we can observe dynamical transitions between conducting and insulating phases as excitons form or ionize on ultrashort timescales.
\end{abstract}

\section{Introduction}

Understanding charge correlations in many-body systems forms a central theme in condensed matter physics. Confinement of carriers in semiconductor nanostructures, in particular, entails enhanced Coulomb correlations and unique low-energy excitations, and offers a well-defined model system to investigate complex interactions between large numbers of quasiparticles. Electrons and holes can form excitons, which exhibit internal transitions between their levels. Small reduced effective masses $\mu$ and large dielectric constants $\varepsilon$ in semiconductors renormalize the Rydberg energy by a factor $\mu /\left(\mathrm{m}_{0} \varepsilon^{2}\right)_{\ll 1}$ and yield exciton binding energies of only a few meV. Whereas generation and annihilation of excitons was extensively explored with near-visible photons, studies of internal exciton transitions - at $\mathrm{THz}$ frequencies orders of magnitude below the optical bandgap - remained scarce. Microscopic theory further supports the notion that $\mathrm{THz}$ absorption of electron-hole gases provides important information about charge correlations, excitons and their dynamics [1]. Pulsed $\mathrm{THz}$ radiation offers a unique tool to measure such transient low-energy excitations. Here, we discuss optical-pump THz-probe experiments that explore dynamical processes of electron-hole gases on a picosecond timescale [2].

\section{Optical-Pump Terahertz-Probe Experiments}

Ultrashort near-infrared pump pulses are used to excite either excitons or unbound electron-hole pairs in GaAs quantum wells. Selective excitation of either species is achieved through spectral shaping of the amplified near-infrared pulses which are derived from a 250-kHz Ti:sapphire regenerative amplifier system. Probe pulses of $\approx 500$ fs duration spanning the range from $0.5-3 \mathrm{THz}$ are generated and detected in thin ZnTe crystals using optical rectification and electro-optic sampling. The sample investigated here was grown by molecular beam epitaxy and consists of ten 14-nm wide GaAs quantum wells separated by 10 -nm wide $\mathrm{Al}_{0.3} \mathrm{Ga}_{0.7}$ As barriers [3]. 

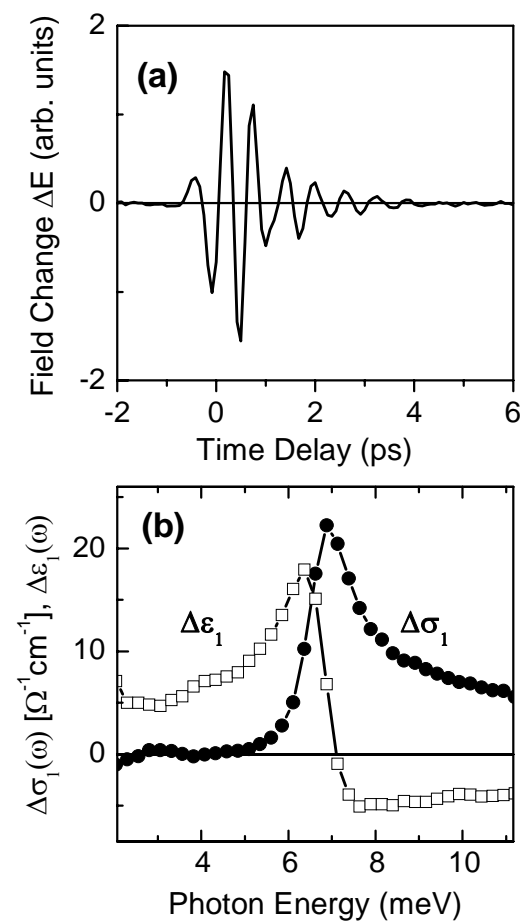

Fig. 1: (a) Induced change $\Delta E(t)$ of the $\mathrm{THz}$ field transmitted through photoexcited GaAs/AlGaAs quantum wells, and (b) corresponding change in the THz conductivity $\Delta \sigma_{1}$ (dots) and dielectric function $\Delta \varepsilon_{1}$ (open squares) at time delay $\Delta \mathrm{t}=5 \mathrm{ps}$ after resonant excitation of $n \approx 10^{10} \mathrm{~cm}^{-2}$ heavy-hole excitons. The data are for lattice temperature $T_{\mathrm{L}}=6 \mathrm{~K}$.

At each pump-probe delay $\Delta t$, we detect the THz field $E(t)$ transmitted through the sample in equilibrium and the pump-induced field change $\Delta E(t)$ shown in Fig 1a. Straightforward electrodynamical relations then yield the transient change of both real and imaginary parts of the complex THz conductivity $\sigma(\omega)=\sigma_{1}(\omega)+i \cdot \sigma_{2}(\omega)$. We take into account the phase shifts in the multilayer structure. In what follows, the response is analyzed in terms of (i) the real part $\sigma_{1}(\omega)$ which yields a measure of absorptive processes and (ii) the dispersive imaginary part, conveniently expressed via the real dielectric function: $\sigma_{2}(\omega)=\omega / 4 \pi\left[1-\varepsilon_{1}(\omega)\right]$. Availability of both parts is central to understanding the transient physical states.

\section{Intra-Exciton Terahertz Response}

A distinct asymmetric peak appears in the THz conductivity $\Delta \sigma_{1}$ around $7 \mathrm{meV}$ photon energy, as shown in Fig 1b, after resonant excitation at the 1s heavy-hole exciton line at low lattice temperature $(\mathrm{T}=6 \mathrm{~K})$. The dispersive feature seen in $\Delta \varepsilon_{1}$ corroborates the appearance of this far-infrared oscillator. Calculations explain the peak which arises from transitions between the exciton bound states, most notably from the $1 \mathrm{~s} \rightarrow 2 \mathrm{p}$ transition. Due to the correlated motion of electrons and holes, charge-neutral excitons are electrically insulating up to a frequency that matches the separation between their lowest internal states. This is reflected directly in the THz conductivity. In contrast, above-bandgap excitation at elevated temperatures (not shown) generates unbound $e$ - $h$ pairs which represent a conducting ionized gas with a Drude-like response.

\section{Dynamics of Conducting and Insulating Phases}

The distinct responses of these extreme phases allows us to follow in time a transition between conducting and insulating phases which occurs upon formation of excitons out of a gas of unbound $e-h$ pairs. Non-resonant excitation above the bandgap is employed to generate unbound e-h pairs at 


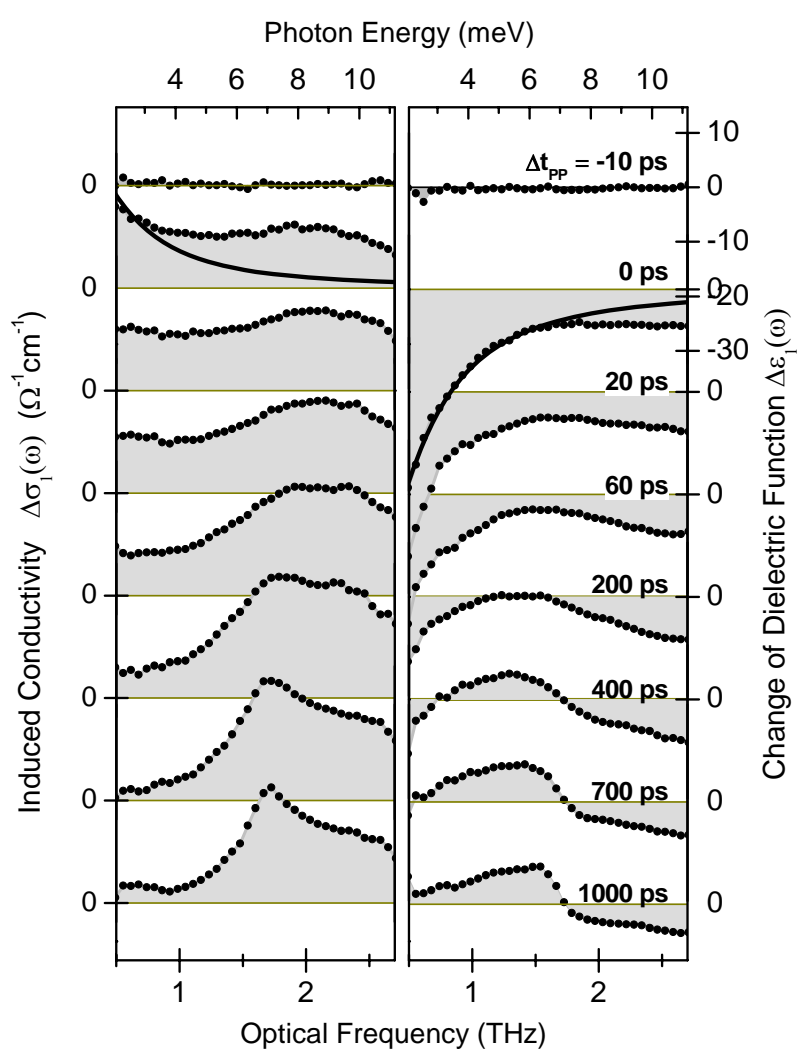

Fig. 2: Transient spectra at $T_{\mathrm{L}}=6 \mathrm{~K}$ after excitation into the continuum of unbound e-h pairs. The excitation pulse is tuned 21 $\mathrm{meV}$ above the $\mathrm{HH} 1 \mathrm{~s}$ exciton line. The thick solid line at $\Delta t=0 \mathrm{ps}$ is a Drude model calculation, and all panels are equally scaled.

$\mathrm{T}=6 \mathrm{~K}$. Fig 2 shows that directly after excitation ( $0 \mathrm{ps})$ we indeed observe a broadband $\mathrm{THz}$ response that indicates a predominantly conducting gas of ionized e-h pairs. Low-frequency conductivity is acquired in $\Delta \sigma_{1}(\omega)$ and the dielectric profile in $\Delta \varepsilon_{1}(\omega)$ changes into the all-negative response expected from a Drude oscillator. However, even at the earliest times, it is important to note that the conductivity is not fully described by the Drude model (thick line) but rather around $\approx 8$ meV photon energy shows a strong excitonic enhancement. With increasing delay time in Fig 2, the spectra exhibit complete reshaping as they evolve into a characteristic exciton response. Binding of pairs into excitons on a timescale of several 100 ps eventually gives rise to an insulating quantum state with fundamental excitation gap equal to the exciton binding energy. The decay of conducting properties is a direct indicator of the increasingly correlated motion of oppositely charged quasi-particles. Two distinct timescales appear in exciton formation, one associated with the quasi-instantaneous appearance of a strong excitonic enhancement, and another much slower transformation from the photoexcited conducting e-h gas to a fully charge-neutral excitonic phase.

\section{Conclusions and Outlook}

In summary, we study fundamental processes of exciton physics by probing internal exciton transitions at $\mathrm{THz}$ frequencies. Resonant generation of excitons results in a low-energy response closely described by transitions between an exciton's internal degrees of freedom. Changes in the $\mathrm{THz}$ response are sensitive indicators for the transient state of an $e-h$ gas. We anticipate that the distinctively different nature of $\mathrm{THz}$ conductivity compared to interband probes will enable new studies of many-body effects and correlated phases of $e$ - $h$ pairs.

\section{References}

[1] M. Kira, W. Hoyer, T. Stroucken, and S. W. Koch "Exciton Formation in Semiconductors and the Influence of a Photonic Environment” Phys. Rev. Lett. vol. 87, 2001, 176401. 
[2] R. A. Kaindl, M. A. Carnahan, D. Hägele, R. Lövenich, and D. S. Chemla. "Ultrafast terahertz probes of transient conducting and insulating phases in an electron-hole gas”, Nature vol. 423, 2003, 734-738, and references therein.

[3] R. Lövenich, C. W. Lai, D. Hägele, D. S. Chemla, and W. Schäfer, "Semiconductor polarization dynamics from the coherent to the incoherent regime: Theory and experiment”, Phys. Rev. B vol. 66, 2002, 045306.

*present address: Institut für Festkörperphysik, Universität Hannover, Appelstraße 2, 30167 Hannover, Germany

Acknowledgements. We thank J. Reno (Sandia Natl. Lab.) for growth of the quantum wells. This work was supported by the Director, Office of Science, Office of Basic Energy Sciences, Division of Materials Sciences, of the US Department of Energy under Contract No. DE-AC03-76SF00098, the Deutsche Forschungsgemeinschaft and the Alexander von Humboldt Foundation.

\section{DISCLAIMER}

This document was prepared as an account of work sponsored by the United States Government. While this document is believed to contain correct information, neither the United States Government nor any agency thereof, nor The Regents of the University of California, nor any of their employees, makes any warranty, express or implied, or assumes any legal responsibility for the accuracy, completeness, or usefulness of any information, apparatus, product, or process disclosed, or represents that its use would not infringe privately owned rights. Reference herein to any specific commercial product, process, or service by its trade name, trademark, manufacturer, or otherwise, does not necessarily constitute or imply its endorsement, recommendation, or favoring by the United States Government or any agency thereof, or The Regents of the University of California. The views and opinions of authors expressed herein do not necessarily state or reflect those of the United States Government or any agency thereof or The Regents of the University of California. 